\title{
Combined Heat and Power Markets by Double-Sided Auction Mechanisms
}

\author{
Roland Saur \\ Centrum Wiskunde \& Informatica (CWI) \\ and Delft University of Technology \\ The Netherlands \\ roland@cwi.nl
}

\author{
Neil Yorke-Smith \\ Delft University of Technology \\ The Netherlands \\ and American University of Beirut \\ n.yorke-smith@tudelft.nl
}

\author{
Han La Poutré \\ Centrum Wiskunde \& Informatica (CWI) \\ and Delft University of Technology \\ The Netherlands \\ Han.La.Poutre@cwi.nl
}

\begin{abstract}
In order to reduce CO2 emissions, energy systems using different energy carriers (e.g., heat and power) are becoming more intertwined and integrated. However, coordination between non-cooperative participants of these systems in the combined heat and power domain has been limited to single-sided auctions with one centralised seller. In this paper, we present a double-sided auction mechanism in which prosumers as well as consumers and producers of heat and power can participate. By showing that our mechanism is Incentive Compatible and Individually Rational, we ensure that truthful bidding is the optimal strategy, simplifying the bidding process and thus accommodating agents with limited computational resources. Finally, we show that our mechanism is fiscally sustainable, i.e., Weakly Budget Balanced.
\end{abstract}

Index Terms-Combined Heat and Power, Double Sided Auctions, Mechanism Design

\section{INTRODUCTION}

Global warming is one of the defining challenges of the 21 st century. In order to reduce $\mathrm{CO} 2$ emissions, significant investments in energy infrastructure and renewable energy sources (RES) have been made. These investments have had profound effects on the energy sector. Excess electricity production from fluctuating RES had to be curtailed at times [1], while heating systems have been switched from gas-fired or electric boilers to combined heat and power plants (CHPs), which convert fuel much more efficiently by producing both heat and electricity.

Closer coordination between power and heat systems promises more efficient use of energy, meaning less curtailment of RES [1, 2], better use of exhaust heat from industrial process [3] and more economic operation of CHPs with District Heating Networks [4]. Attempts of better integration between heat and power have often focused on how to give individual CHPs the flexibility to react to fluctuating renewable electricity production, by considering heat storage - either dedicated $[4,5]$ or indirectly in District Heating Networks [6] - or by extending the operational range through the use of electric boilers [4, 5]. Zhong et al. [7] (with some extensions in [8]) introduced a multi-energy auction mechanism to coordinate between self-interested agents. However, this line of work to date is limited to single-sided auctions in which participants only buy electricity and heat from a single centralised seller.
We address the problem of facilitating trade between several producers and consumers across the heat and power domain. Our results are as follows. We first develop a market model that allows both consumers and producers as well as power-toheat devices to participate. We then design a double auction mechanism for allocating supply and demand based on trade reduction [9] for this market model. We show that our mechanism incentivises agents to participate truthfully in the market, i.e., the mechanism is Individually Rational (IR) [10] and Incentive Compatible (IC) [10]. We thereby reduce the need for the agents to strategize. Lastly we prove that our market design does not require subsidies, i.e., the mechanism is Weakly Budget Balanced (WBB) [11].

\section{THE MODEL}

We introduce our market model and define desirable properties for pricing mechanisms. Every agent $l$ in the set of agents $N$ has a desired bundle $\left(i_{l}, j_{l}\right)$, with $i_{l}, j_{l} \in\{0,1,-1\}$, and associates a private valuation $v_{l} \in \mathbb{R}$ with this bundle. $i$ indicates the amount of electricity while $j$ is the amount of heat. Positive values in the bundle indicate consumption, while negative values are associated with production. E.g., a bundle $(1,-1)$ means an agent wants to consume one electricity and produce one heat. Every agent $l \in N$ submits one bid $b_{l}$ consisting of its desired bundle and a reserve price $\rho_{l} \in \mathbb{R}$ : $b_{l}=\left(\left(i_{l}, j_{l}\right), \rho_{l}\right)$. The reserve price is the maximum price an agent is willing to pay for its bundle, with negative values seen as payments to the agents. Agents posses a quasi-linear utility function $u_{l}(X, P)$, where $X$ is the binary allocation vector, with $x_{l}=1$ if agent $l$ is allocated and $x_{l}=0$ otherwise, and $P_{\left(i_{l}, j_{l}\right)}$ is the price set for bundle $\left(i_{l}, j_{l}\right)$ by the mechanism. Not receiving any bundle leaves the agent with zero utility.

$$
u_{l}(X, P)=v_{l} \cdot x_{l}-P_{\left(i_{l}, j_{l}\right)} \cdot x_{l}
$$

An allocation is feasible if it results in material balance, i.e., $\sum_{l \in N} i_{l} \cdot x_{l}=0 \wedge \sum_{l \in N} j_{l} \cdot x_{l}=0$, ensuring that every unit of energy produced is also consumed within the system.

We assume a disinterested market operator concerned with maximising the social welfare of the system as a whole. Similarly to [12], we define social welfare as the sum of all agents (Trader and Auctioneer). The utility of the auctioneer is the surplus the mechanism generates. We can write the social 
welfare of an allocation as $S W=\sum_{l} u_{l}+u_{\text {auctioneer }}=$ $\sum_{l}\left(v_{l}-P_{\left(i_{l}, j_{l}\right)}\right) x_{l}+\sum_{l} P_{\left(i_{l}, j_{l}\right)} x_{l}=\sum_{l} v_{l} x_{l}$.

Assumption 1: All agents report their true valuation: $\rho_{l}=v_{l}$. With the above assumption, we can express our task as follows:

$$
\begin{aligned}
& S W=\sum_{l \in N} \rho_{l} x_{l} \\
& \text { s.t. } \sum_{l \in N} i_{l} \cdot x_{l}=0 ; \quad \sum_{l \in N} j_{l} \cdot x_{l}=0 \\
& x_{l} \in\{0,1\},
\end{aligned}
$$

where the first constraint ensures strict material balance, while the second constraint prohibits partial allocation of bundles.

\section{A. Properties Relevant to Mechanism Design}

In this section, we will justify our assumption of truthful bidding, $\rho_{l}=v_{l}$, see Assumption 1. To coordinate between all the bidding agents, a mechanism takes in all bids and outputs an allocation of trading agents and prices. However, as the mechanism only receives reported bids $\left(\rho_{l}\right)$ and not true valuations $\left(v_{l}\right)$, it only maximizes social welfare if the agents report their private valuations truthfully.

Mechanisms that are Incentive Compatible (IC) make truthful reporting the utility maximising strategy for every agent independent of the behaviour of other agents. Assumption 1 is, therefore, fulfilled under the much weaker assumption of utility maximising agents if the mechanism is IC. Further, making truthful reporting the optimal strategy independent of the bids of other agents obviates the need for sophisticated bidding strategies. This enables small agents with limited computational power to efficiently operate in the market. Additionally, to make participation appealing to agents, the mechanism should be Individually Rational (IR), i.e., reporting truthfully to the auctioneer never leads to negative utility for the reporting agent.

However, the seminal paper by Myerson and Satterthwaite [13] showed that it is impossible to design a mechanism that is IC, IR, optimal in social welfare and Weakly Budget Balanced (WBB) at the same time, meaning any optimal, IC and IR mechanism necessarily runs a budget deficit, making it unsustainable. This impossibility result can be amended by trade reduction mechanisms, which artificially raise buying and lower selling prices by removing trading agents, in effect sacrificing some social welfare for WBB [9].

The formal definitions of these concepts are as follows:

Definition 1 (IC): [10] A mechanism is IC if for all agents truthfully reporting their valuation maximises their utility independent of what others bid.

Definition 2 (IR): [10] A mechanism is IR, if for all agents truthful reporting never leads to negative utility.

Definition 3 (WBB): [11] A mechanism is WBB, if the payments by the agents to the mechanism always outweigh the payments made by the mechanism.

We strive to develop a mechanism that is IC, IR and WBB.

\section{TRAdE REDUCTION MECHANISM}

In this section, we introduce our trade reduction mechanism. We make the following two assumptions.
Assumption 2: No two bids have the exact same valuation.

Assumption 3: The market is deep: there is a sufficient number of bids per bundle allocated.

Our mechanism outputs both a final allocation and a set of prices, one for each bundle. As described in Alg. 1, it computes the optimal allocation $X^{*}$ (line 1), removes the lowest two bids per bundle (lines 5 and 6), and sets the prices to the value reported by the removed bids (line 4).

Input: Set of Bids $B$

Output: Final allocation $X^{\square}$ and prices $P$

1: $X^{*} \leftarrow \operatorname{argmax}_{X} S W(X, B)$

2: for $i \in\{0,1,-1\}$ do

3: $\quad$ for $j \in\{0,1,-1\}$ do

4: $\quad P_{(i, j)} \leftarrow \rho_{s(i, j)}$

5: $\quad x_{k(i, j)}^{*} \leftarrow 0$

6: $\quad x_{s(i, j)}^{*} \leftarrow 0$

7: $P \leftarrow \bigcup_{i, j \in\{0,1,-1\}} P_{(i, j)}$

8: $X^{\square} \leftarrow X^{*}$

9: return $\left(X^{\square}, P\right)$

Alg. 1. Trade reduction mechanism returns the final allocation and prices.

To describe the process in more detail, we introduce $k(i, j)$ as the allocated agent with the lowest bid for bundle $(i, j)$ : $k(i, j)=\operatorname{argmin}_{m \in N}\left\{\rho_{m} \mid\left(i_{m}, j_{m}\right)=(i, j) \wedge x_{m}^{*}=1\right\}$.

Next to $k(i, j)$, we define the next lowest bid for every bundle as $s(i, j)$.

$$
s(i, j)=\underset{m \in N}{\operatorname{argmin}}\left\{\rho_{m} \mid \rho_{m}>\rho_{k(i, j)},\left(i_{m}, j_{m}\right)=(i, j)\right\}
$$

The price for bundle $(i, j)$ is defined by agent $s(i, j)$.

$$
P_{(i, j)}=\rho_{s(i, j)}, \quad i, j \in\{0,1,-1\}
$$

\section{A. General Properties}

Before we discuss the desired properties of our trade reduction mechanism (i.e., IR, IC, WBB), we establish some properties that are required for the proofs in Section III-B. Notably, we establish that all agents in the final allocation reported a value above the price defining agent (see Property 2 ), remain allocated as long as they report a value above the value of the price defining agent (see Property 7) and cannot influence the price while allocated (see Property 8).

First we introduce some notation. Let $N^{*}$ be the set of all agents allocated under allocation $X^{*}: N^{*}=\left\{l \in N \mid x_{l}^{*}=1\right\}$.The set of all agents interested in bundle $(i, j)$ is denoted as $N(i, j)=\left\{l \in N \mid\left(i_{l}, j_{l}\right)=(i, j)\right\}$.

The two agents, $k(i, j)$ and $s(i, j)$, which are removed by the trade reduction mechanism, will be denoted as $N^{\Delta}(i, j)$. This set is clearly defined because of Assumptions 2 and 3 .

$$
\begin{gathered}
\quad N^{\Delta}(i, j) \subset N^{*}(i, j) \\
\text { s.t. }\left|N^{\Delta}(i, j)\right|=2 \wedge\left(\rho_{m}>\rho_{l}\right) \\
\forall l \in N^{\Delta}(i, j), \forall m \in N^{*}(i, j) \backslash N^{\Delta}(i, j)
\end{gathered}
$$

Agent $s(i, j)$ is therefore the highest valued bid in $N^{\Delta}(i, j)$.

$$
s(i, j)=\underset{l}{\operatorname{argmax}}\left\{\rho_{l} \mid l \in N^{\Delta}(i, j)\right\}
$$


The set of all removed bids is defined as $N^{\Delta}=$ $\bigcup_{(i, j)} N^{\Delta}(i, j)$, which allows us to describe the final allocation $N^{\square}$ as follows:

$$
N^{\square}=N^{*} \backslash N^{\Delta} \text {. }
$$

We consider an agent $l \in N$ interested in bundle $(i, j)$ and consider the effects of only agent $l$ changing its bid. For this, we introduce the bidding profile $b_{-l}=\left(b_{1}, b_{2}, \ldots b_{l-1}, b_{l+1}, \ldots b_{n}\right)$ as the set of bids from all agents except for agent $l$. We define $N^{1 *}\left(\left((i, j), \rho_{l}^{1}\right), b_{-l}\right)$ and $N^{2 *}\left(\left((i, j), \rho_{l}^{2}\right), b_{-l}\right)$ as the optimal allocations resulting from agent $l$ bidding $\rho_{l}^{1}$ and $\rho_{l}^{2}$ under bidding profile $b_{-l}$, respectively. For brevity, we will write $N^{1 *}$ and $N^{2 *}$ instead of $N^{1 *}\left(\left((i, j), \rho_{l}^{1}\right), b_{-l}\right)$ and $N^{2 *}\left(\left((i, j), \rho_{l}^{2}\right), b_{-l}\right)$.

We show that an allocated agent cannot affect the optimal allocation by increasing its bid (see Property 1 and Corollary 1) and describe all allocated agents (see Property 2).

Property 1: Given a bidding profile $b_{-l}$ and an agent $l$ interested in bundle $(i, j)$ the following statement holds:

$$
\left(\rho_{l}^{2}>\rho_{l}^{1} \wedge l \in N^{1 *}\right) \Longrightarrow N^{1 *}=N^{2 *} \text {. }
$$

Proof: Assume by contradiction that $\rho_{l}^{2}>\rho_{l}^{1} \wedge l \in N^{1 *}$ and $N^{1 *} \neq N^{2 *}$. We define $T^{\Delta}=N^{1 *} \backslash N^{2 *}$ and $T^{-\Delta}=$ $N^{2 *} \backslash N^{1 *}$, with which we can express $N^{2 *}$ as follows:

$$
N^{2 *}=\left(N^{1 *} \backslash T^{\Delta}\right) \cup T^{-\Delta} .
$$

As $N^{2 *}$ is the optimal allocation given bids $B^{2}=$ $\left(\left((i, j), \rho_{l}^{2}\right), b_{-l}\right)$, we know that $\sum_{m \in N^{2 *}} \rho_{m}^{2} \geq \sum_{m \in N^{1 *}} \rho_{m}^{2}$. Applying equation (8) gets us to the following expression:

$$
\begin{gathered}
\sum_{m \in N^{1 *}} \rho_{m}^{2}-\sum_{m \in T^{\Delta}} \rho_{m}^{2}+\sum_{m \in T^{-\Delta}} \rho_{m}^{2} \geq \sum_{m \in N^{1 *}} \rho_{m}^{2} \\
\sum_{m \in T^{-\Delta}} \rho_{m}^{2}-\sum_{m \in T^{\Delta}} \rho_{m}^{2} \geq 0 .
\end{gathered}
$$

Given the fixed bidding profile $b_{-l}$ and $\rho_{l}^{2}>\rho_{l}^{1}$, we know that $\rho_{m}^{2} \geq \rho_{m}^{1} \quad \forall m \in N$ and therefore $\sum_{m \in T} \rho_{m}^{2} \geq$ $\sum_{m \in T^{\Delta}} \rho_{m}^{1}$. Furthermore, $\rho_{m}^{1}=\rho_{m}^{2}$ holds for all $m \neq l$ from which follows that $\sum_{m \in T^{-} \Delta} \rho_{m}^{2}=\sum_{m \in T^{-} \Delta} \rho_{m}^{1}$ as $l \notin T^{-\Delta}$. Applying these insights to equation (9) results in:

$$
\begin{gathered}
\sum_{m \in T^{-\Delta}} \rho_{m}^{1}-\sum_{m \in T^{\Delta}} \rho_{m}^{1} \geq \sum_{m \in T^{-\Delta}} \rho_{m}^{2}-\sum_{m \in T^{\Delta}} \rho_{m}^{2} \geq 0 \\
\sum_{m \in T^{-\Delta}} \rho_{m}^{1}-\sum_{m \in T^{\Delta}} \rho_{m}^{1} \geq 0
\end{gathered}
$$

There exist two cases for equation (10) - strictly larger and equality. When $\sum_{m \in T^{-\Delta}} \rho_{m}^{1}-\sum_{m \in T^{\Delta}} \rho_{m}^{1}>0$ holds, equation (10) contradicts our assumption that $N^{1 *}$ is optimal, as $N^{1 *} \backslash T^{\Delta} \cup T^{-\Delta}$ results in higher social welfare. When equality holds, either $T^{-\Delta}=T^{\Delta}=\varnothing$ in which case $N^{1 *}=N^{2 *}$ or there exist multiple optimal allocations, one of which is $N^{2 *}$; consistent tie breaking will lead to $N^{1 *}=N^{2 *}$.

Corollary 1: Given a bidding profile $b_{-l}$ and an agent $l$ the following holds: $\left(l \in N^{1 *} \wedge l \in N^{2 *}\right) \Longrightarrow N^{1 *}=N^{2 *}$.

Property 2 (Allocated Agents):

$$
\rho_{l}>\rho_{s(i, j)} \quad \forall l \in N^{\square}(i, j)
$$

Proof: $s(i, j) \in N^{\Delta}(i, j)$. The result follows from the definitions of $N^{\Delta}(i, j)$ and $N^{\square}(i, j)$ in (5) and (7).
We next introduce the central concept of a trade (see Definition 4) as the building block of allocations (see Property 3 ) and show that trades can at most contain 2 bids on the same bundle (see Properties 4 and 5).

Definition 4 (Trade): A trade $T$ is a nonempty set of agents $m$ s.t. $\left(\sum_{m \in T} i_{m}=0 \wedge \sum_{m \in T} j_{m}=0\right)$ while $\nexists \tilde{T} \subsetneq T \quad$ s.t. $\quad \tilde{T} \neq \varnothing \wedge \sum_{m \in \tilde{T}} i_{m}=0 \wedge \sum_{m \in \tilde{T}} j_{m}=0$

Property 3: Every feasible allocation set $N^{X}$ can be partitioned into a set of trades: $N^{X}=\cup_{m} T_{m}$

Proof: Take any feasible allocation set $N^{X}$. Since $N^{X}$ is feasible we know that $\left(\sum_{m \in N^{X}} i_{m}=0 \wedge \sum_{m \in N^{X}} j_{m}=0\right)$. One of two cases applies. $N^{X}$ can be partitioned into two feasible non-empty allocation sets, which can be partitioned again. $N^{X}$ cannot be partitioned into two feasible allocation sets. Therefore there does not exist a non-empty strict subset of $N^{X}$ that is feasible and $N^{X}$ is a trade by Def. 4 .

Property 4: No trade $T$ can contain more than one bid for the same complex bundle $(i, j) i, j \neq 0$.

Proof: The proof goes to show that assuming the opposite always leads to a trade within a trade, which contradicts our definition of a trade. We start by assuming that there is a trade $T$ and two bids $l, m \in T$ for a bundle $(i, j) i, j \neq 0$. Let $T^{-}=T \backslash\{l, m\}$ be the residual trade. For $T$ to be materially balanced $\sum_{r \in T^{-}} i_{r}=-2 i \wedge \sum_{r \in T^{-}} j_{r}=-2 j$ has to hold. Therefore, $T^{-}$needs to contain at least two bids on bundles $\{(-i, 0),(-i,-j),(-i, j)\}$ and at least two bids on bundles $\{(0,-j),(-i,-j),(i,-j)\}$. However, if there exists a bid $r \in T^{-}$on bundle $(-i,-j)$, then $\{r, l\} \subsetneq T$ is a trade. Similarly there cannot exist two bids $r_{1}, r_{2} \in T^{-}$on bundles $(-i, 0)$ and $(0,-j)$, as then $\left\{r_{1}, r_{2}, l\right\} \subsetneq T$ is a trade. Furthermore, there cannot be two bids $r_{1}, r_{2} \in T$ on bundles $(-i, j)$ and $(i,-j)$, since $\left\{r_{1}, r_{2}\right\} \subsetneq T$ would constitute a trade. Therefore $T^{-}$is either exclusively composed of bids on bundles $\{(-i, 0),(i,-j)\}$ or of bids on $\{(0,-j),(-i, j)\}$. We will only look at the case of $T^{-}$consisting of bids on bundles $\{(-i, 0),(i,-j)\}$, as the other case follows similarly. Recall that $\sum_{r \in T^{-}} i_{r}=-2 i \wedge \sum_{r \in T^{-}} j_{r}=-2 j$ has to hold for $T^{-}$ and therefore its bids are on the following bundles $[4 \cdot(-i, 0), 2$. $(i,-j)]$. However then $T=T^{-} \cup\{l, m\}$ consists of bids with bundles $[4 \cdot(-i, 0), 2 \cdot(i,-j), 2 \cdot(i, j)]$, which can be split into two feasible trades of the form $[2 \cdot(-i, 0),(i,-j),(i, j)]$, contradicting our assumption that $T$ is a trade.

Property 5: No trade can contain more than two bids for the same simple bundle $(i, j) \in\{(1,0),(0,1),(-1,0),(0,-1)\}$

Proof: We assume, contrary to the above property, that there exists a trade $T$ with bids $l, m, o \in T$ on a bundle $(i, 0)$, $i \in\{1,-1\}$. Let $T^{-}=T \backslash\{l, m, o\}$. For $T$ to be feasible $\sum_{r \in T^{-}} i_{r}=-3 i$ and therefore there have to be three bids on bundles $\{(-i, 0),(-i, 1),(-i,-1)\}$. However, there cannot be a bid $r \in T$ on bundle $(-i, 0)$. Otherwise $\{r, l\} \subsetneq T$ and $T \backslash\{r, l\}$ are feasible trades, contradicting our definition of a trade. Therefore, there have to be 3 bids on bundles in $\{(-i, 1),(-i,-1)\}$, which means that at least two bids are on the same complex bundle which violates Property 4 . This contradiction holds similarly for 3 bids on a bundle $(0, j)$, $j \in\{1,-1\}$ 
With this knowledge about the composition of trades, we establish the value of the price defining agent as a lower bound on what an allocated agent could have bid and still be allocated (see Properties 6 and 7).

Property 6: Given a bidding profile $b_{-l}$, an agent $l$ interested in bundle $(i, j)$ and its bid $\left((i, j), \rho_{l}^{1}\right)$ such that $l \in N^{1 *}$ :

$$
\rho_{l}^{2}>\rho_{s^{1}(i, j)} \Longrightarrow l \in N^{2 *} \text {. }
$$

Proof: Given an agent $l$ with bid $\left((i, j), \rho_{l}^{1}\right)$ such that $l \in N^{1 *}$, assume contrary to the property that $\rho_{l}^{2}>\rho_{s^{1}(i, j)}$ and $l \notin N^{2 *}$. There are two cases to be considered.

Case 1: $l \in\left\{s^{1}(i, j), k^{1}(i, j)\right\}$ : If so, we know that $\rho_{l}^{2}>$ $\rho_{s^{1}(i, j)} \geq \rho_{l}^{1}$ and thus $l \in N^{2 *}$ by Property 1 . This contradicts the initial assumption.

Case 2: $l \notin\left\{s^{1}(i, j), k^{1}(i, j)\right\}$ : Since, by the initial assumption, $l \notin N^{2 *}$ and $\rho_{l}^{2}>\rho_{s^{1}(i, j)}>\rho_{k^{1}(i, j)}$, it follows that $s^{1}(i, j), k^{1}(i, j) \notin N^{2 *}$. Otherwise $N^{2 *}$ could be improved by swapping $k^{1}(i, j)$ or $s^{1}(i, j)$ for $l$.

We will arrive at a contradiction by showing that $N^{1 *}$ is not optimal when $l$ bids $\rho_{l}^{1}$. Define $T^{\Delta}=N^{1 *} \backslash N^{2 *}$ and $T^{-\Delta}=N^{2 *} \backslash N^{1 *}$. Since $l, s^{1}(i, j), k^{1}(i, j) \in N^{1 *}$ and $l, s^{1}(i, j), k^{1}(i, j) \notin N^{2 *}$ we know that $l, s^{1}(i, j), k^{1}(i, j) \in$ $T^{\Delta}$. Consider improving $N^{1 *}$ by identifying a balanced exchange of agents occurring in the process of going from $N^{1 *}$ to $N^{2 *}$ that is independent of agent $l$.

First, we express the removal of $T^{\Delta}$ and addition of $T^{-\Delta}$ as a new hypothetical allocation. To do so, we introduce $M^{\Delta}$ as a mirror image of $T^{\Delta}$ as follows. For every agent $m \in$ $T^{\Delta}$ define one corresponding agent $m^{M} \in M^{\Delta}$ with bid $b_{m^{M}}=\left(\left(-i_{m},-j_{m}\right),-\rho_{m}\right)$. Let $k^{M}, s^{M}, l^{M} \in M^{\Delta}$ be the corresponding agents for agents $k^{1}(i, j), s^{1}(i, j), l \in T^{\Delta}$, respectively. $\left(M^{\Delta} \cup T^{-\Delta}\right)$ is a feasible allocation, disjoint from $N^{1 *}$, that represents the exchange of agents when going from $N^{1 *}$ to $N^{2 *}$.

Second, to show that $N^{1 *}$ can be improved upon, we identify a trade in $\left(M^{\Delta} \cup T^{-\Delta}\right)$ that is independent of $l$ as follows. Let $T_{k} \subseteq\left(M^{\Delta} \cup T^{-\Delta}\right)$ be a maximum value trade such that $k^{M} \in T_{k}$. We know that $l^{M} \notin T_{k}$. Otherwise it would follow from Properties 4 and 5, and $l^{M}, k^{M} \in T_{k}$, that $s^{M} \notin T_{k}$. Then, since $-\rho_{s^{1}(i, j)}>-\rho_{l}, T_{k} \backslash\left\{l^{M}\right\} \cup\left\{s^{M}\right\}$ would have a higher value, contradicting our definition of $T_{k}$ as a maximum value trade.

Third, we now use $T_{k}$ to improve upon $N^{1 *}$, as follows. Let $T_{k}^{-\Delta}=T_{k} \cap T^{-\Delta}$ and let $T_{k}^{\Delta} \subseteq T^{\Delta}\left(\subset N^{1 *}\right)$ be the set of agents that correspond to the agents in the mirror image $T_{k} \cap M^{\Delta}$. As $N^{2 *}$ is optimal, $N^{1 *} \backslash T_{k}^{\Delta} \cup T_{k}^{-\Delta}$ has a higher social welfare than $N^{1 *}$, when $l$ bids $\rho_{l}^{2}$. However, since $l^{M} \notin$ $M^{\Delta}$ and therefore $l \notin T_{k}^{\Delta}, N^{1 *} \backslash T_{k}^{\Delta} \cup T_{k}^{-\Delta}$ has a higher social welfare than $N^{1 *}$ independent of what $l$ bids, contradicting our assumption that $N^{1 *}$ is optimal when $l$ bids $\rho_{l}^{1}$.

Property 7: Given a bidding profile $b_{-l}$, an agent $l$ interested in bundle $(i, j)$ and its bid $\left((i, j), \rho_{l}^{1}\right)$ such that $l \in N^{1 \square}$ :

$$
\rho_{l}^{2}>\rho_{s^{1}(i, j)} \Longrightarrow l \in N^{2 \square} \text {. }
$$

Proof: As $l \in N^{1 \square}$ and $N^{1 \square} \subset N^{1 *}$, we know that $l \in N^{1 *}$. Given $l \in N^{1 *}$ and $\rho_{l}^{2}>\rho_{s^{1}(i, j)}$ it follows by Property 6 that $l \in N^{2 *}$ and thus $N^{1 *}=N^{2 *}$, see Corollary 1 . By our initial assumption, we know that $\rho_{l}^{2}>\rho_{s^{1}(i, j)}>\rho_{k^{1}(i, j)}$ and by $N^{1 *}=N^{2 *}$ we know that $s^{1}(i, j), k^{1}(i, j) \in N^{2 *}$. This implies that $l$ is not one of the lowest two bids on bundle $(i, j)$ in $N^{2 *}$, i.e., $l \notin N^{2 \Delta}$. From $l \notin N^{2 \Delta} \wedge l \in N^{2 *}$ it follows that $l \in N^{2 \square}$.

Further, we show that agents in the final allocation cannot at the same time affect the price they pay for their bundle and remain in the final allocation (see Property 8 ).

Property 8: Given a bidding profile $b_{-l}$ and an agent $l$ interested in bundle $(i, j)$ :

$$
l \in N^{1 \square} \wedge l \in N^{2 \square} \Longrightarrow P_{(i, j)}^{1}=P_{(i, j)}^{2} .
$$

Proof: Recall that $s(i, j)$ is the price defining agent for bundle $(i, j)$ (see Equation (4)) and therefore it suffices to show that $\rho_{s^{1}(i, j)}=\rho_{s^{2}(i, j)}$. Since $N^{\square} \subset N^{*}$, it follows from our initial assumption that $l \in N^{1 *} \wedge l \in N^{2 *}$ and therefore $N^{1 *}=N^{2 *}$, by Corollary 1. As $l \in N^{1 \square} \wedge l \in N^{2 \square}$, we know that $l \notin N^{1 \Delta} \wedge l \notin N^{2 \Delta}$. Since all bids of agents other than $l$ are fixed by $b_{-l}$, we know that $N^{1 \Delta}=N^{2 \Delta}$ and by extension $\rho_{s^{1}(i, j)}=\rho_{s^{2}(i, j)}$.

\section{B. Mechanism design relevant properties}

With the above general properties in place, we can now show that our trade reduction mechanism (Alg. 1) satisfies all three desired properties: IR, IC and WBB (see section II).

1) Individual Rationality (IR): To ensure IR, we need to show that agents truthfully reporting their valuation can never obtain negative utility.

Theorem 1 (Individual Rationality): The mechanism is Individually Rational, i.e., $u_{l}\left(X\left(\rho_{l}=v_{l}\right), P\left(\rho_{l}=v_{l}\right)\right) \geq 0$

Proof: Assume that trading agent $l$ has a bid $b=\left((i, j), \rho_{l}\right)$ and true valuation $v_{l}$. If agent $l$ is not allocated $l \notin N^{\square}$, then its utility is 0 . If agent $l$ is allocated $l \in N^{\square}$, its utility is $u_{l}=v_{l}-P_{(i, j)}=v_{l}-\rho_{s(i, j)}=\rho_{l}-\rho_{s(i, j)} \geq 0$. The inequality holds because of Property 2. For the auctioneer agent IR holds due to WBB, see Theorem 3.

2) Incentive Compatible(IC): To show that the mechanism satisfies IC, we need to show that no agent can strictly increase its utility by bidding anything but its true valuation.

Theorem 2 (Incentive Compatibility): The mechanism is Incentive Compatible, i.e. $u_{l}\left(\rho_{l}=v_{l}\right) \geq u_{l}\left(\rho_{l} \neq v_{l}\right) \quad \forall l \in N$.

Proof: To show that no agent can improve its utility by deviating from bidding its true valuation, we separately examine agents that would and would not be allocated under truthful bidding. Let agent $l$ have private valuation $v_{l}$ and let $\rho_{l}^{1}=v_{l}$. Case $l \in N^{1 \square}$ (allocated under truthful bidding):

There are two cases to be considered for the bid of agent $l$. Either it is allocated or not when bidding $\rho_{l}^{2}$.

- $l \in N^{2 \square}$ : Through property 8 , we know that $l \in N^{1 \square} \wedge l \in$ $N^{2 \square}$ implies $P_{(i, j)}^{1}=P_{(i, j)}^{2}$ and the utility of the agent can be written as $u_{l}\left(\rho_{l}^{2}\right)=v_{l}-P_{(i, j)}^{2}=v_{l}-P_{(i, j)}^{1}=$ $u_{l}\left(\rho_{l}^{1}=v_{l}\right)$.

- $l \notin N^{2 \square}$ : Given $l \notin N^{2 \square}$, agent $l$ receives zero utility and its utility is: $u_{l}\left(\rho_{l}^{2}\right)=0 \leq u_{l}\left(\rho_{l}^{1}=v_{l}\right)$. The inequality holds because the mechanism is Individually Rational.

Case $l \notin N^{1 \square}$ (not allocated under truthful bidding):

There are two cases to be considered for the bid of agent $l$. Either it is allocated or not when bidding $\rho_{l}^{2}$. 
- $l \in N^{2 \square}$ : Recalling that $s(i, j)$ is the price defining agent, the utility of agent $l$ can be written as $u_{l}\left(\rho_{l}^{2}\right)=$ $v_{l}-\rho_{s^{2}(i, j)} \leq 0 \leq u\left(\rho_{l}^{1}=v_{l}\right)$. Indeed, we know that $v_{l} \leq \rho_{s^{2}(i, j)}$ as otherwise $\rho_{l}^{1}=v_{l}>\rho_{s^{2}(i, j)}$ leads to a contradiction: By Property 7 (index reversed), $\rho_{l}^{1}>\rho_{s^{2}(i, j)}$ and $l \in N^{2 \square}$ implies that $l \in N^{1 \square}-$ a contradiction. The second inequality holds because the mechanism is Individually Rational.

- $l \notin N^{2 \square}$ : As both $l \notin N^{1 \square}$ and $l \notin N^{2 \square}$, it follows that the utility of agent $l$ is zero either way.

3) Weak Budget Balance (WBB): To ensure WBB, we need to show that payments made to the mechanism are larger than payments made by the mechanism.

Theorem 3 (Weak Budget Balance): The mechanism is weakly budget balanced.

Proof: Given Property 3, we can split any allocation $N^{\square}$ into trades and therefore only have to show that no trade can run a budget deficit. The budget of a trade $T \subset N^{\square}$ can be written as the sum of the prices associated with the bundles of the agents in the trade. Recall that the price for a bundle $\left(i_{m}, j_{m}\right)$ is set by the corresponding agent $s\left(i_{m}, j_{m}\right) \in N^{*}$, see equation (4). Alternatively, we can sum over all possible bundles $(i, j)$ and multiply each price by the number of times the bundle occurs in $T$. For this, let $\left|T_{(i, j)}\right|$ be the number of agents in $T$ that bid on bundle $(i, j)$. Then:

$$
\begin{aligned}
\operatorname{Budget}(T) & =\sum_{m \in T} P_{\left(i_{m}, j_{m}\right)}=\sum_{m \in T} \rho_{s\left(i_{m}, j_{m}\right)}=\sum_{(i, j)} \rho_{s(i, j)}\left|T_{(i, j)}\right| \\
& \geq \sum_{(i, j)}\left\{\begin{array}{ll}
\rho_{s(i, j)}+\rho_{k(i, j)} & \text { if }\left|T_{(i, j)}\right|=2 \\
\rho_{s(i, j)} & \text { if }\left|T_{(i, j)}\right|=1 \\
0 & \text { if }\left|T_{(i, j)}\right|=0
\end{array} \geq 0 .\right.
\end{aligned}
$$

By Properties 4 and 5 , we know that $\left|T_{(i, j)}\right|$ can only be 0 , 1 or 2 . By the way we defined $s(i, j)$, see (3), we know that $2 \cdot \rho_{s(i, j)}>\rho_{s(i, j)}+\rho_{k(i, j)}$ and therefore the first inequality holds. The sum that follows in the second line sums over the valuations of a trade $T_{s, k}$ that is contained in $N^{*} . T_{s, k}$ has the same bundle composition as the original trade $T$ but consists exclusively of agents $s(i, j)$ and $k(i, j)$. Since we assumed $N^{*}$ to be optimal in Social Welfare, the sum of the valuations of any trade in $N^{*}$ has to yield a non-negative value and therefore the second inequality holds.

\section{CONClusion AND Future Work}

This paper addressed combined heat and power markets. We developed a market setting in which not only consumers and producers, but also agents that want to buy one commodity and simultaneously sell another, e.g. power-to-heat devices, can participate. We introduced a trade reduction mechanism which does not require subsidies and which incentivises agents to participate truthfully, creating an efficient marketplace in which agents can participate without the need for sophisticated bidding strategies. By removing the computational burden from individual agents, we enable a future energy system to coordinate between small self-interested energy devices.
Allowing bids on arbitrary bundles and accommodating more than two energy carriers are future extensions of this work.

\section{ACKNOWLEDGMENT}

This work is part of the research programme Heat and Power Systems at Industrial Sites and Harbours (HaPSISH) with project number OND1363719, which is partly financed by the Dutch Research Council (NWO).

\section{REFERENCES}

[1] D. Böttger, M. Götz, N. Lehr, H. Kondziella, and T. Bruckner, "Potential of the power-to-heat technology in district heating grids in Germany," Energy Procedia, vol. 46, pp. 246-253, 2014.

[2] S. Mueller, R. Tuth, D. Fischer, B. Wille-Haussmann, and C. Wittwer, "Balancing fluctuating renewable energy generation using cogeneration and heat pump systems," Energy Technology, vol. 2, no. 1, pp. 83-89, 2014.

[3] S. H. Chae, S. H. Kim, S.-G. Yoon, and S. Park, "Optimization of a waste heat utilization network in an eco-industrial park," Applied Energy, vol. 87, no. 6, pp. 1978-1988, 2010.

[4] I. Dimoulkas and M. Amelin, "Constructing bidding curves for a CHP producer in day-ahead electricity markets," in Energy Conf. (ENERGYCON), 2014, pp. 487-494.

[5] X. Chen, C. Kang, M. O’Malley, Q. Xia, J. Bai, C. Liu, R. Sun, W. Wang, and H. Li, "Increasing the flexibility of combined heat and power for wind power integration in China: Modeling and implications," IEEE Trans. Power Systems, vol. 30, no. 4, pp. 1848-1857, 2015.

[6] Z. Li, W. Wu, J. Wang, B. Zhang, and T. Zheng, "Transmission-constrained unit commitment considering combined electricity and district heating networks," IEEE Trans. on Sust. Energy, vol. 7, no. 2, pp. 480-492, 2016.

[7] W. Zhong, K. Xie, Y. Liu, C. Yang, and S. Xie, "Auction mechanisms for energy trading in multi-energy systems," IEEE Trans. Industr. Inform., vol. 14, no. 4, pp. 1511$1521,2018$.

[8] W. Zhong, C. Yang, K. Xie, S. Xie, and Y. Zhang, "Admm-based distributed auction mechanism for energy hub scheduling in smart buildings," IEEE Access, vol. 6, pp. $45635-45645,2018$.

[9] R. P. McAfee, "A dominant strategy double auction," Journal of Economic Theory, vol. 56, no. 2, pp. 434 450, 1992.

[10] N. Nisan, T. Roughgarden, E. Tardos, and V. V. Vazirani, Algorithmic Game Theory. Cambridge Univ. Press, 2007.

[11] R. K. Dash, N. R. Jennings, and D. C. Parkes, "Computational-mechanism design: A call to arms," IEEE Intelligent Systems, vol. 18, pp. 40-47, 2003.

[12] L. Y. Chu, "Truthful bundle/multiunit double auctions," Management Science, vol. 55, no. 7, pp. 1184-1198, 2009.

[13] R. B. Myerson and M. A. Satterthwaite, "Efficient mechanisms for bilateral trading," Journal of Economic Theory, vol. 29, no. 2, pp. 265-281, 1983. 